EOMmUn: Communication et organisation

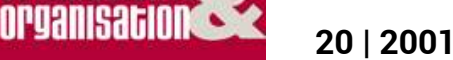

La communication du risque

Opération « silhouette »

Georges Aubert

CpenEdition

Journals

Édition électronique

URL : http://journals.openedition.org/communicationorganisation/2573

DOI : 10.4000/communicationorganisation.2573

ISSN : 1775-3546

Éditeur

Presses universitaires de Bordeaux

Édition imprimée

Date de publication : 1 novembre 2001

ISSN : 1168-5549

Référence électronique

Georges Aubert, "Opération « silhouette » », Communication et organisation [En ligne], 20 | 2001, mis en ligne le 27 mars 2012, consulté le 01 mai 2019. URL : http://journals.openedition.org/ communicationorganisation/2573; DOI : 10.4000/communicationorganisation.2573

Ce document a été généré automatiquement le 1 mai 2019.

(C) Presses universitaires de Bordeaux 


\title{
Opération « silhouette »
}

\author{
Georges Aubert
}

1 Pour le moins que l'on puisse l'écrire, la problématique du déplacement en sécurité est complexe et comme l'ont montré Alain Gras, Caroline Moricot, Sophie L. Poirot-Delpech... [et al.] (1994), l'erreur humaine est la manifestation d'un ensemble de petites erreurs produites par le système socio-technique, d'emboîtements, et ou de défaillances successives inattendues. Ces défaillances peuvent résulter de l'écart entre les représentations de l'usager et les représentations de l'ingénieur. Ceci sous-entend que théoriquement les systèmes proposés par les concepteurs doivent être préparés à l'erreur. L'usager est faillible et le niveau de complexité de son mode de déplacement est facteur de risque. Nous approchons ainsi la notion de risque, et plus particulièrement de prise de risque passive qui s'impose à l'usager (Le Breton, 1991), i.e., une prise de risque qu'il n'a ni préparée, ni souhaitée, ni rêvée. Les prises de risque passives sont les conséquences d'inadaptations que les usagers n'identifient pas comme telles et que l'utilisation ou la référence au sens commun ne permet pas de lever. Complémentairement (Le Breton, 1991), la prise de risque active est préparée, souhaitée, voire rêvée par l'usager. Pour les sociologues, le goût du risque relèverait d'une spécificité des sociétés occidentales où il est devenu un moyen de formation et d'intégration sociale. Le risque y est défini comme une relation imaginaire ou réelle à la mort, les fonctions du risque permettant à l'individu de légitimer de sa présence, d'arracher le sentiment d'exister, de se sentir physiquement contenu et enfin d'être assuré dans son identité (Le Breton, 1991). Ainsi, la prise de risque serait un processus par lequel le sujet donne du sens à son existence et la valorise. Il va donc interroger différents signifiants dont un ultime « la mort », l'approche de la mort nourrissant un sentiment d'identité renouvelée et générant du sens. L'alternative au risque c'est la sécurité qui est un autre processus qui permet de dire la signification et la valeur de l'existence. Se comporter en sécurité c'est prévoir, c'est s'assurer de ses propres anticipations. L'orientation est donc plus altruiste. Il s'agit de moins se mettre en avant, de s'épargner, i.e., de ne pas aller sur ses propres limites et donc de rester en retrait sur soi-même. Afin de mieux neutraliser toute situation hasardeuse, précaire ou étrangère. 
2 En psychologie du développement, les faits établis privilégient à la fois une approche conceptuelle et procédurale des phénomènes psychologiques (Karlmiloff-Smith, 1992). Communiquer sur le risque doit tenir compte de ce double aspect du fonctionnement cognitif. Dans la démarche de communication, on pourra privilégier un aspect ou l'autre.

3 Les difficultés que pose l'approche conceptuelle: On pourrait dire qu'il y a autant de conception du déplacement et de la prise de risque dans le déplacement qu'il y a d'usagers. En fait, lorsqu'on étudie les conceptions ${ }^{1}$ (Johsua et Dupin, 1993) des enfants dans des domaines comme les mathématiques (Ponce 1993, 1996), la logique (Aubert, 1994, 1998), les sciences naturelles (Ponce 2000), etc. Les différentes conceptions que l'on rencontre peuvent être catégorisées en quelques collections.

4 Les difficultés posées par une approche procédurale du risque sont liées au fait qu'une procédure est fortement dépendante des contextes d'utilisation ou d'apprentissage et qu'il faudrait donc multiplier les contextes afin de couvrir une réalité la plus large possible.

5 La recherche d'un outil de communication sur le risque routier qui répond à plusieurs critères :

6 - simplicité (tant du point de vue de l'exploitant que de l'usager),

7 - capacité de déclenchement (i.e. susceptible d'agir sur les usagers selon leur conception du risque dans le déplacement),

8 - socialement acceptable (i.e. acceptable par les usagers qui ont été victimes du risque routier),

9 - répondre à des objectifs de sécurité routière (institutionnels), s'est orientée vers la recherche ou la conception d'un symbole. Ce symbole doit être un signifiant qui permette d'agir sur les différentes conceptions des usagers. Ce symbole doit répondre à une contradiction, être le plus dépouillé possible pour agir sur les différentes conceptions tout en étant le plus évocateur possible. Or, comme nous l'avons écrit précédemment, ce qui est ultime dans la prise de risque c'est une interrogation de la mort. Il s'est agi, alors, d'analyser dans notre société l'existant qui traite du lien entre «la prise de risque routier » et la mort. Notre société est porteuse de nombreuses associations et nous avons découvert qu'une association du département de l'Hérault ${ }^{2}$ avait mené en collaboration avec la coordinatrice sécurité routière de ce département une opération consistant à disposer des silhouettes sur les lieux d'accident. Cette opération n'avait pas été évaluée. Nous avons très rapidement compris l'intérêt de ce symbole, il serait socialement acceptable (car proposé par un parent de victime de la route), il signifie au minimum la mort (référant ultime susceptible d'être compris par tous), la mort « signifié » susceptible d'agir sur les usagers.

10 Partant, le préfet de la préfecture de Gironde a initié l'opération appelée "Silhouette », opération dont l'originalité réside essentiellement en la volonté de reprendre l'action qui s'était déroulée dans l'Hérault et de l'accompagner par une évaluation menée par un organisme indépendant le $\mathrm{LaCo}^{3}$. Cette action formalisait une initiative de l'état au plan local (Mission sécurité routière de la préfecture de Gironde) et répondait aux orientations définies pour la campagne d'intérêt public de la sécurité routière qui avait été décrétée grande cause nationale pour l'année 2000. Deux orientations sont distinguées: la sensibilisation, l'information et la formation d'une part et les activités innovantes en faveur de la sécurité routière d'autre part. 
11 Cette opération utilise de simples silhouettes noires (dépouillées de tout graphisme) comme outil de communication et vise plusieurs objectifs : d'une part, prévenir le risque par l'information et la dissuasion, et, d'autre part, faire réagir l'usager.

Il s'agit, en quelque sorte, d'essayer d'apporter à l'usager des informations susceptibles d'amorcer un échange susceptible de faire baisser la violence routière observée dans les activités de déplacements. Ainsi, des silhouettes ont été placées sur les lieux d'accidents en nombre égal au nombre de tués. Seul le réseau national connaissait ce dispositif (RN10, RN89, RN113, RN137, RN215, RN250), aucune silhouette n'était disposée sur les autres chaussées du réseau routier en Gironde.

13 A priori, l'observation sur le terrain montre que des silhouettes ont été vandalisées (arrachées, décorées). Ces comportements signifient-ils que les silhouettes ont choqué l'usager? Ces réactions de l'usager sont celles que l'on pouvait le moins attendre (car non constructives) mais craindre le plus.

14 C'est dans ce contexte que notre travail de recherche s'inscrit. L'objet de ce travail est l'évaluation de la qualité de l'opération «Silhouette» qui nécessite d'interroger, de mettre à plat, les représentations et les attributions élaborées par l'usager.

Pour évaluer la qualité de l'opération, deux aspects sont à aborder, l'action en qualité de vecteur d'information (qualité communicative) et l'action en qualité de contenu d'information (qualité de communication). Ainsi, nous distinguons: La qualité communicative, i.e. l'impact et la facilité de transmission; La qualité de communication, i.e. le contenu des informations véhiculées par les usagers.

En somme, il s'agit d'évaluer la qualité de cet outil de communication tant du point de vue du vecteur que des contenus d'information qu'il peut véhiculer.

Pour répondre à ces nécessités des objectifs multiples sont à réaliser :

18 - Mesurer l'impact de l'opération sur la population Girondine ;

19 - Mesurer la qualité de la transmission (selon que l'on s'intéresse à l'usager en qualité d'émetteur, ou de récepteur d'informations) ;

20 - Déterminer les représentations suggérées chez l'usager.

21 - Mettre au point un outil d'évaluation qui permet la réalisation des objectifs précédents.

22 Nous allons présenter dans un premier temps, l'outil d'évaluation qui a été mis au point. Ensuite, nous présenterons la méthode de recueil des données. Pour finir, les résultats de cette étude seront exposés.

\section{Opérationnalisation}

\section{L'outil d'évaluation}

23 Nous allons définir dans un premier temps l'esprit de l'évaluation. L'objectif de ce travail est de réunir des éléments qui permettent d'identifier les représentations des personnes qui ont pu être en contact avec les silhouettes. Il ne s'agit ni d'enregistrer un discours académique ou convenu sur cette problématique, ni pour autant de rechercher l'originalité. Ce qui nous intéresse, c'est le simple vécu des personnes vis-à-vis des silhouettes. Il faut donc se donner les garanties nécessaires pour obtenir un travail le plus objectif possible. La recherche d'objectivité sera gagnée en contrôlant plusieurs facteurs : 
mis en place d'un outil qui normalise le recueil des données, contrôler les biais possibles qui peuvent être générés par les personnalités des enquêteurs.

Le meilleur moyen identifié pour obtenir des réponses «sincères » des usagers, i.e. des réponses «non académiques" est la mise en place d'une situation construite sur le modèle d'une entrevue. La dynamique de l'entrevue, si elle est bien menée, doit permettre d'obtenir les objectifs préalablement cités. L'objectivité sera renforcée dans la mesure où un cadre de référence sera utilisé pour mener les entrevues. Un questionnaire a donc été réalisé à cet effet, ce questionnaire comprend des questions semis ouvertes, i.e. si l'usager ne parvient pas à répondre à une question ouverte un ensemble de réponses types lui est proposé (plusieurs choix étant possibles pour une même question). Le questionnaire occupait une page d'une feuille de format A4.

Le questionnaire a été conçu de telle façon que l'usager soit progressivement placé dans le contexte du déplacement. Car, nous savons que les connaissances que nous utilisons dans la construction de représentations sont contextualisées. Les premières questions centrent donc l'usager sur son identité sous l'angle de celui qui utilise différentes modalités de déplacement et ont vocation à favoriser un engagement dans l'acte de communication. L'usager est donc questionné sur son âge, sexe, lieu de résidence, profession, permis possédés, ses déplacements, les types de véhicules utilisés, le nombre de kilomètres parcourus.

Après cette mise en condition, les questions suivantes vont interroger les usagers sur la localisation des silhouettes (ont-elles été vues et « où »?), sur leurs impressions, sur leurs réactions, sur les objectifs qu'ils attribuent à cette opération, sur l'organisateur de cette opération, sur leur jugement en qualité d'utilité et d'attente.

Un mode d'emploi qui explique l'esprit et l'utilisation du questionnaire a été établi et fourni aux enquêteurs (questionnaire et mode d'emploi consultables sur le site www.montaigne.u-bordeaux.fr/GRECO).

\section{Méthode}

636 usagers ont été entretenus, l'entretien était conduit avec le questionnaire construit à cet usage (ibid précédemment). Les entretiens ont été réalisés par des inspecteurs départementaux de sécurité routière et des adjoints de sécurité de la mission sécurité routière qui se présentaient en qualité d'enquêteur réalisant une enquête pour le compte $\mathrm{du}$ LaCo/CNRS qui est le laboratoire « Langage et Cognition » associé CNRS de Poitiers. Le questionnaire a été préalablement expliqué aux enquêteurs et est accompagné d'une notice explicative. Sur chaque questionnaire est précisé le nom de l'enquêteur et le lieu de l'enquête.

Les lieux, dans lesquels les entrevues ont été réalisées, répondaient à un souci de représentativité de la population Girondine et de certaines populations concernées à différents titres par les déplacements motorisés. Les lieux d'enquête étaient répartis entre le centre des cartes grises de la préfecture de gironde, des entreprises (CITRAM, EADS, ESG, etc.), des hypermarchés (Libourne), dans différentes villes (Saint-Loubès, Bernos-Beaulac etc.). La population prise en entrevue se caractérise socioprofessionnellement comme suit : 


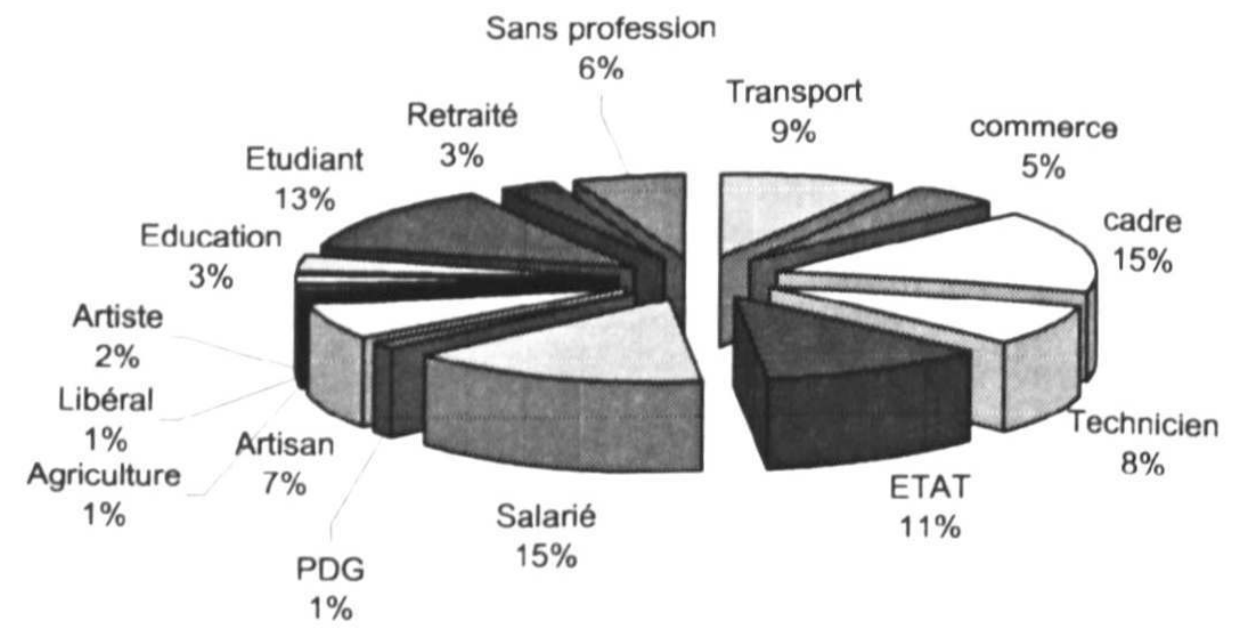

Répartition des usagers selon leur catégorie socio-professionnelle d'égal à égal. Ce devait être l'occasion d'un échange, il fallait donc éviter les situations où il y aurait eu deux enquêteurs face à un enquêté. Le questionnaire était rempli par l'enquêteur qui notait les réponses des usagers.

\section{Résultats :}

\section{Qualité communicative} représentations et la réalité du terrain) ;

38 - Les conditions dans lesquelles les silhouettes auraient été vues ;

39 - Le nombre de personnes indirectement informées (impact);

40 - La qualité de la transmission.

41 - Les silhouettes ont été vues par 86,5\% des usagers interrogés. Les lieux verbalisés par les usagers se répartissent comme suit : 


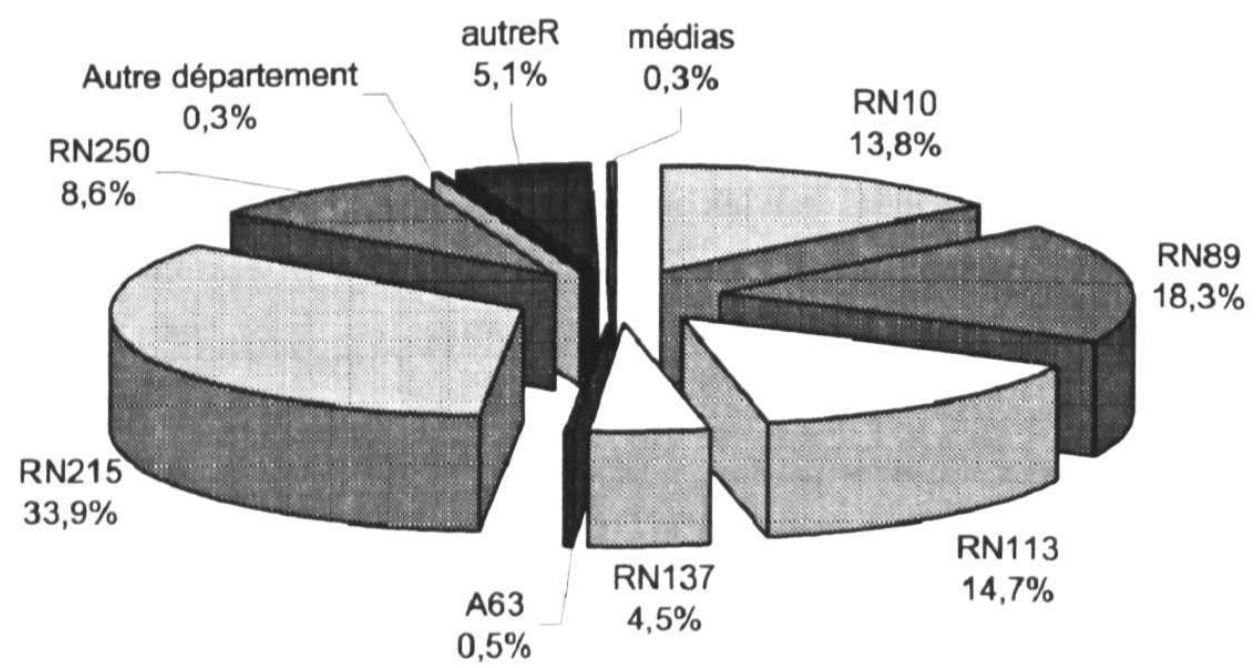

Répartition des lieux verbalisés par les usagers

Fréquence des lieux verbalisés :

Fréquences des lieux verbalisés par les usagers

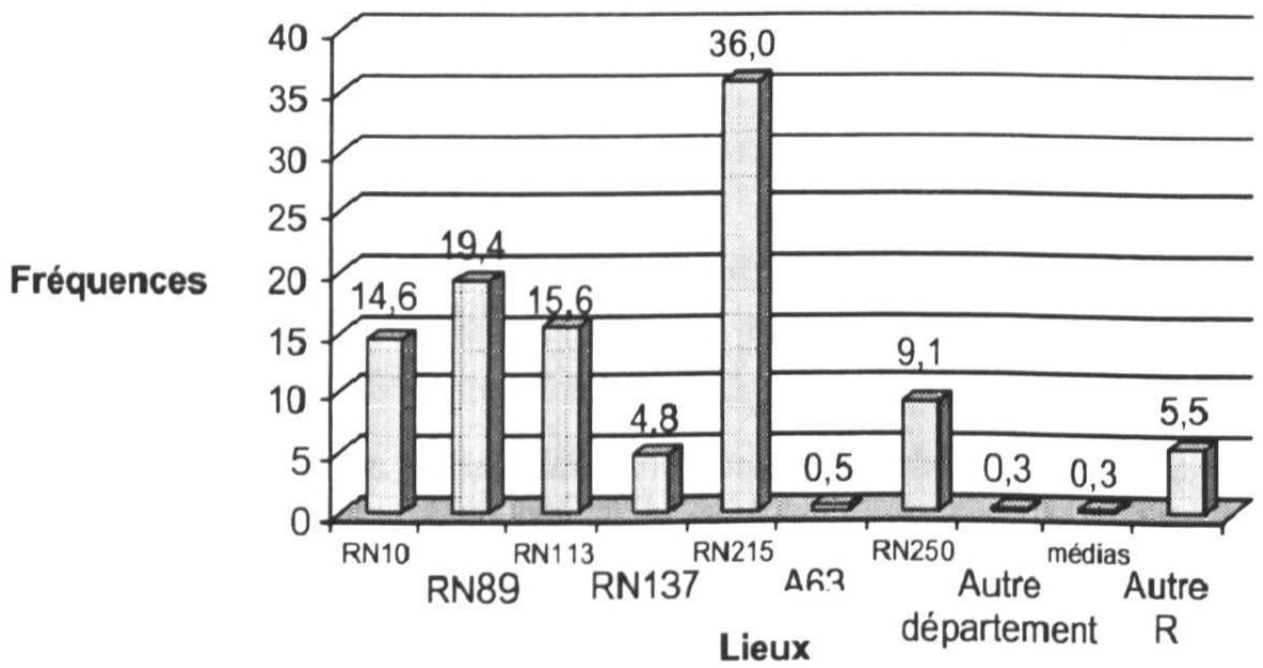

Pour plus de $5 \%$ des usagers, les silhouettes étaient disposées sur toutes les routes du département (réponse "Autre $\mathrm{R} »)$. Il s'agit d'un phénomène de généralisation abusive (sur-généralisation). On peut craindre que les usagers infèrent que les routes départementales sont moins dangereuses que les routes nationales. Ce dernier point fait craindre un biais sur l'effet escompté par l'action dans le sens où les usagers seraient surconfiants sur le réseau routier départemental. Élargir cette action à tout le réseau départemental permet de répondre à ce risque.

Le graphique suivant répartit les conditions de déplacement dans lesquelles les silhouettes ont été vues. 


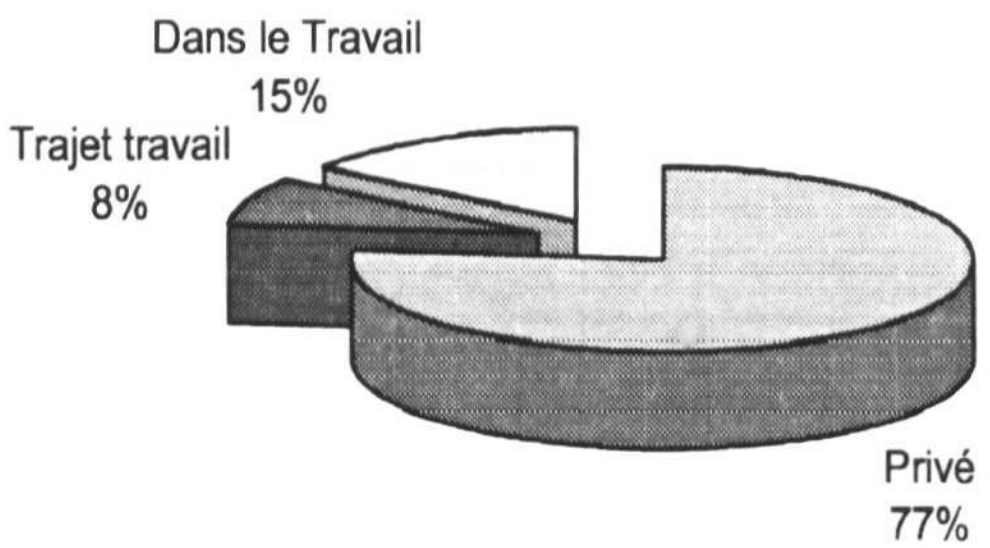

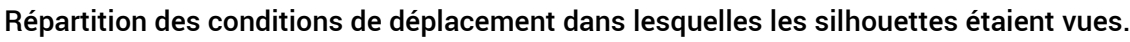

- Les lieux où l'on parle des silhouettes se répartissent comme suit :

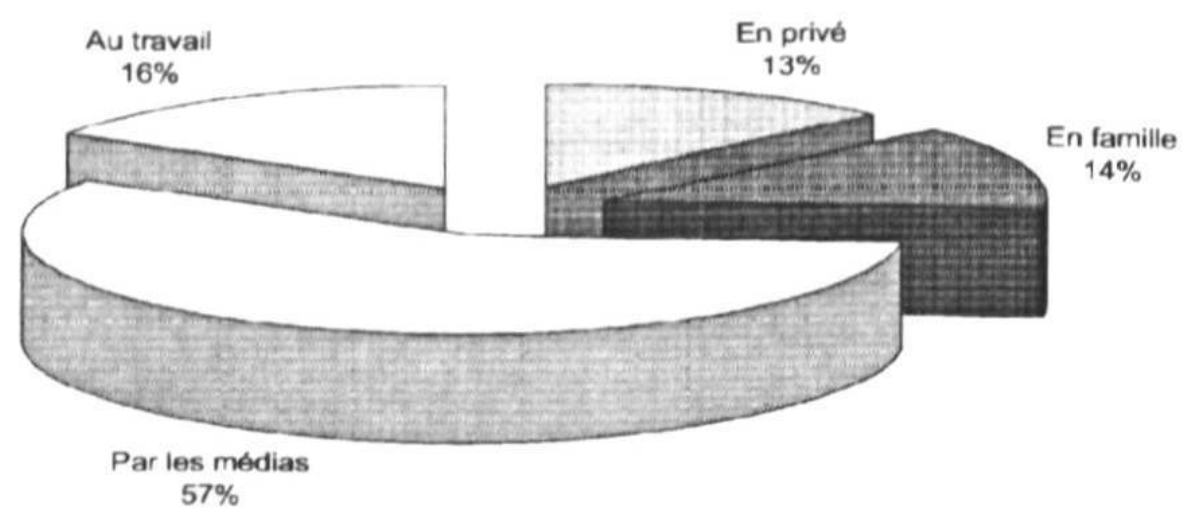

Répartition des lieux où l'on parle des silhouettes

Fréquences des lieux où l'on parle des silhouettes : 


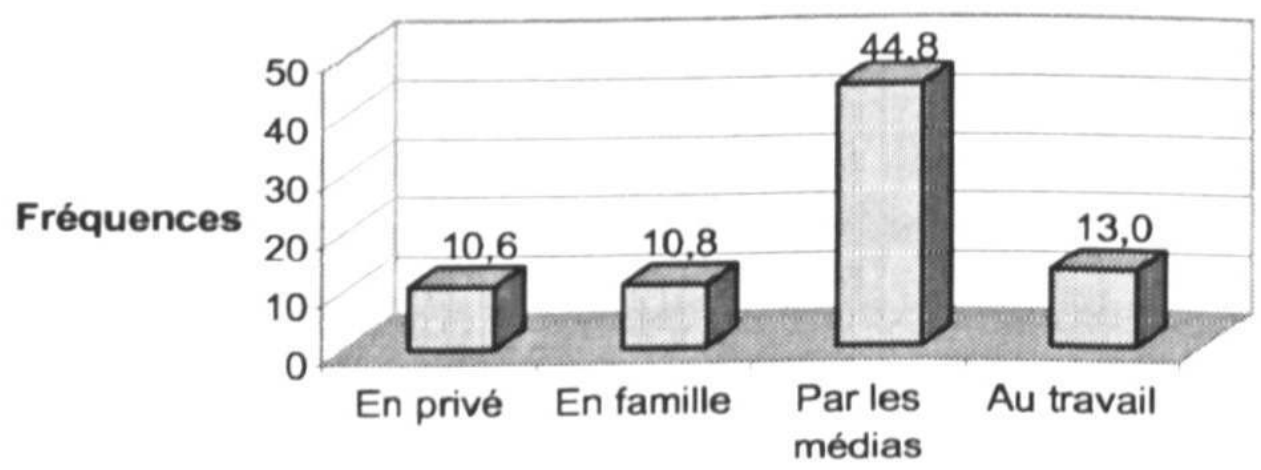

Lieux

Fréquences des lieux où l'on parle des silhouettes équivalent à l'ensemble des autres moyens de communication identifiés.

\section{Qualité de communication:}

61 L'évaluation de la qualité de communication a été réalisée selon plusieurs aspects :

62 - Les impressions suscitées par la vue des silhouettes;

63 - Les réactions engendrées par la vue des silhouettes;

64 - Les objectifs que l'usager attribue à cette action ;

65 - L'organisateur que l'usager attribue pour cette action ;

66 - L'intérêt que l'usager porte sur cette action ;

67 - Les attentes de l'usager pour cette action. 


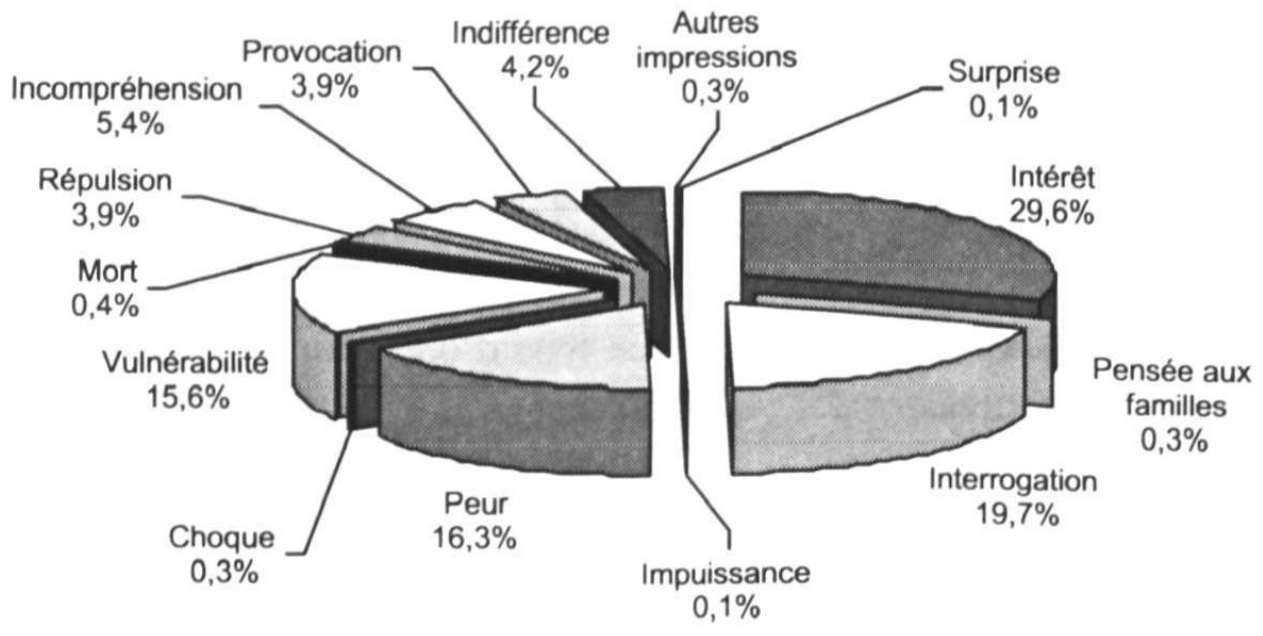

Répartition des impressions verbalisées par les usagers

Fréquences des impressions suscitées par la vue des silhouettes chez les usagers :

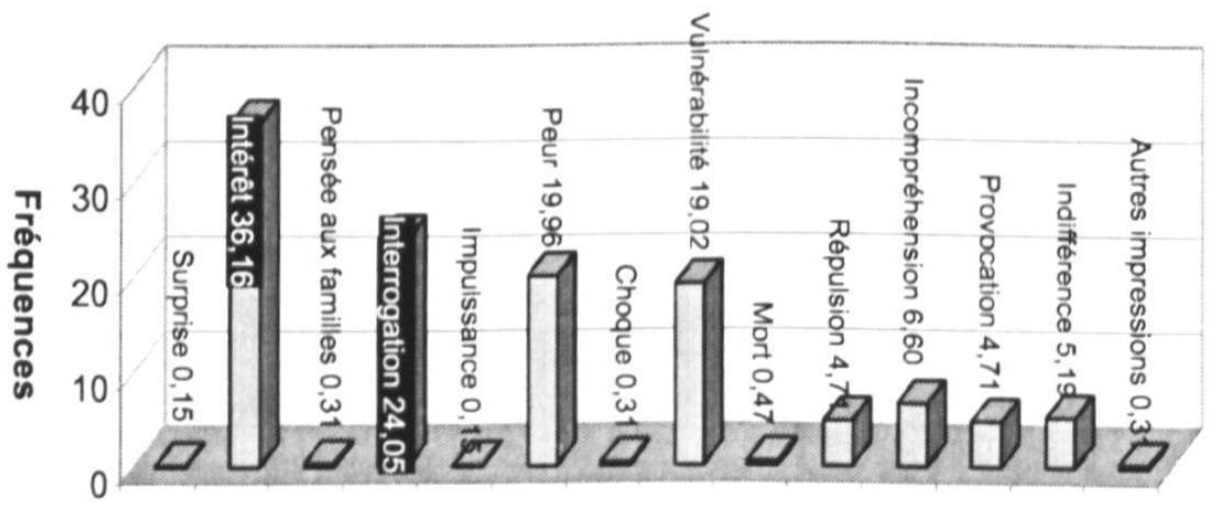

Fréquences des impressions verbalisées par les usagers

L'analyse de ces résultats montre que très peu d'usagers sont choqués par cette opération (3\%). Les actes de vandalisme qui ont pu être observés à rencontre des silhouettes ne révèlent pas un état de choc des usagers. Quatre impressions sur quatorze sont fortement majoritaires (« intérêt » 36,2\%, « interrogation » 24,1\%, " peur » $20 \%$, « vulnérabilité » $19 \%)$. L'intérêt et l'interrogation sont suscités chez les deux tiers des usagers. Ces impressions montrent que cette opération éveille de la curiosité chez l'usager. Plus du tiers des usagers exprime de la peur ou de la vulnérabilité. L'analyse des données par catégories d'usagers ("victime ", " lié à une victime ", " motard ", " usager utilisant un véhicule pendant l'activité de travail») montre que $0,5 \%$ des usagers utilisant un véhicule pendant le travail sont choqués contre $0 \%$ pour les autres catégories. Il semblerait qu'un contact répété avec les silhouettes par la curiosité suscitée gêne l'activité de déplacement car l'usager ne sait pas comment investir cette curiosité et d'autant plus fortement que le déplacement est contraint par le travail. Cette gêne choque certains professionnel de la route.

Les réactions consécutives à la vue des silhouettes se répartissent comme suit : 


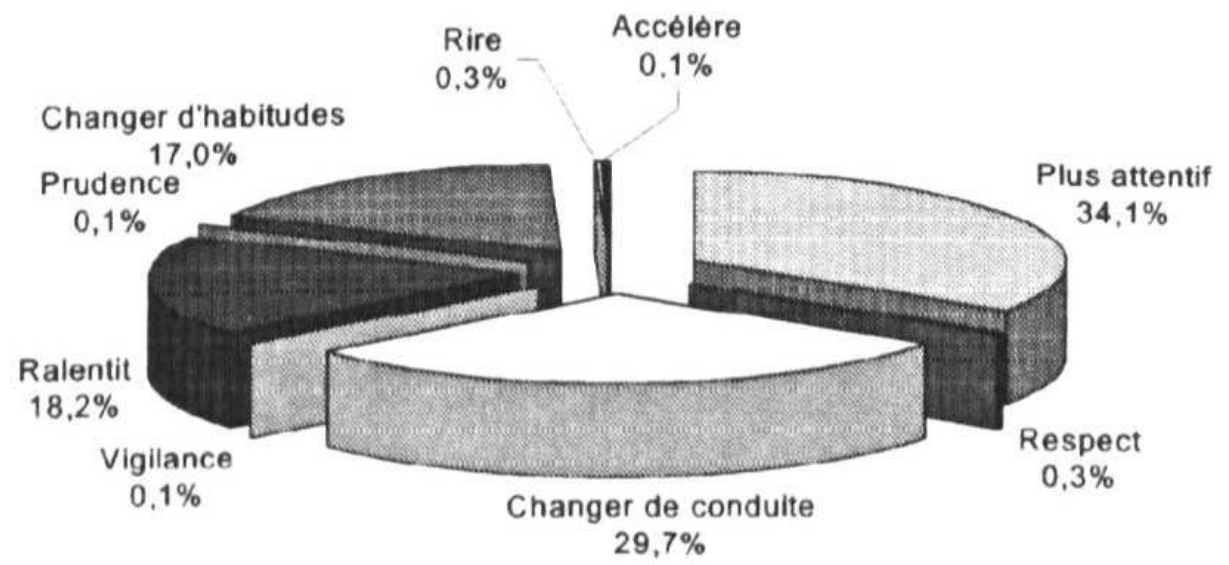

Répartition des réactions verbalisées par les usagers

Fréquences des réactions consécutives à la vue des silhouettes :

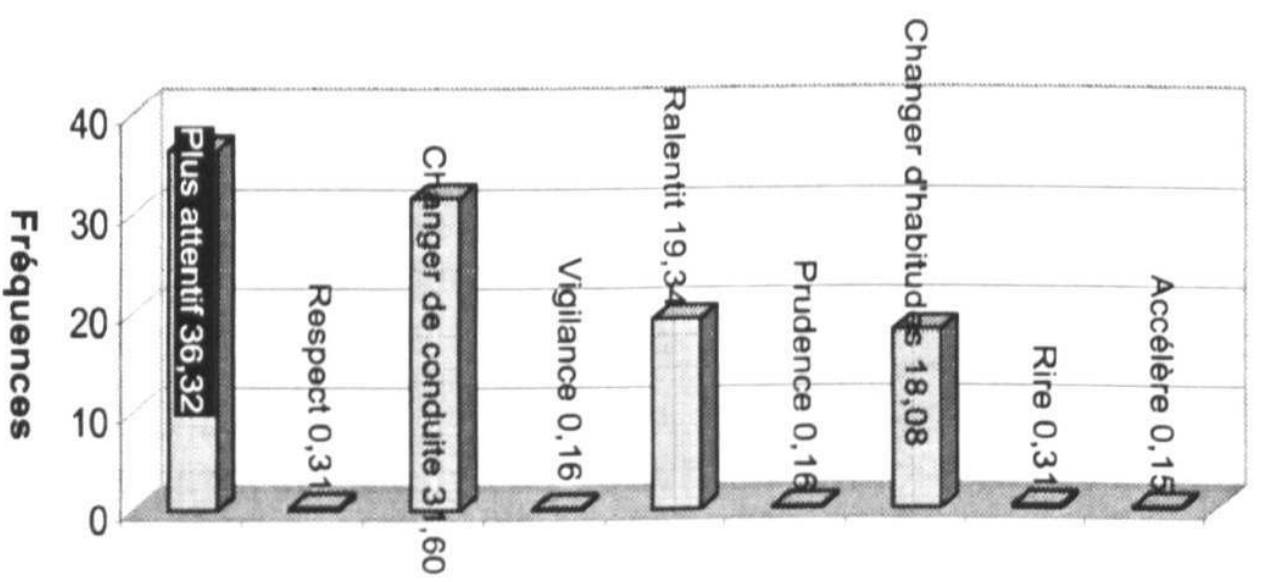

Fréquences des réactions verbalisées par les usagers

L'analyse des résultats montre que $39,2 \%$ des usagers reconnaissent ne pas changer de conduite ou d'habitude. Quatre réactions parmi neuf réactions verbalisées sont fortement majoritaires («être plus attentif» $36,2 \%$, «ne pas changer de conduite» $31,6 \%$, « ralentir » 19,3\%, «ne pas changer d'habitude» $18,1 \%$ ). Ne rien changer aux habitudes est donc la réaction majoritaire observée. Cependant, $36,3 \%$ des usagers reconnaissent être plus attentifs et $19,3 \%$ des usagers disent ralentir. Si les usagers ne changent pas d'habitude, c'est qu'ils sont convaincus de bien conduire ou de réaliser l'acte de conduire convenablement. La vue des silhouettes sur le réseau routier national aiguillonne leur curiosité et ne sachant pas comment l'investir, à défaut d'information, émerge un doute sur la sécurité de ce réseau qu'ils compensent en augmentant leur vigilance ou par défaut en ralentissant. Ce doute affecte leurs croyances sur l'acte de conduire de différentes façons : Remise en question de sa propre capacité à bien conduire qui se traduit par la recherche d'information que l'usager n'aurait pas traitée jusqu'alors (développement des schèmes de conduite); l'usager fait plus attention ou ralentit pour traiter cognitivement d'autres sources d'information. 
ennaissance de situation dont la dangerosité potentielle échappe à toute analyse rationnelle et dont il faut se méfier. L'usager fait plus attention ou ralentit parce qu'il ne sait pas quoi faire d'autre. En d'autre terme que faire contre la fatalité (et si le ciel nous tombait sur la tête !) ?

Les objectifs que les usagers attribuent à l'opération « silhouette » se répartissent comme suit :

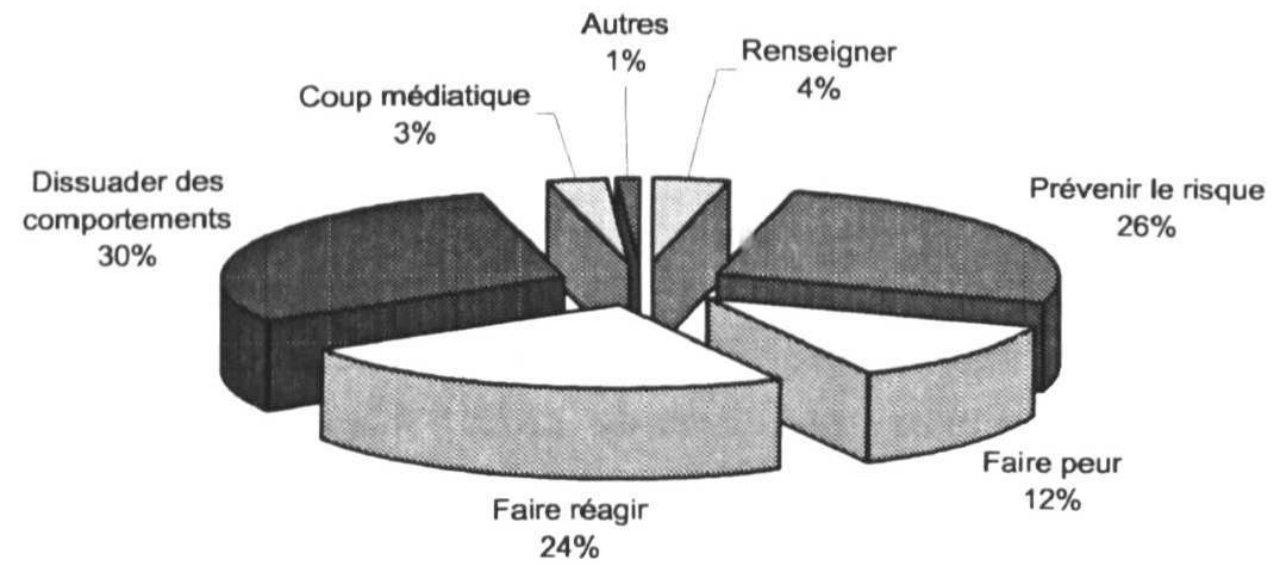

Répartition des objectifs attribués à l'opération par les usagers

Fréquences des objectifs attribués à l'opération « silhouette » par les usagers :

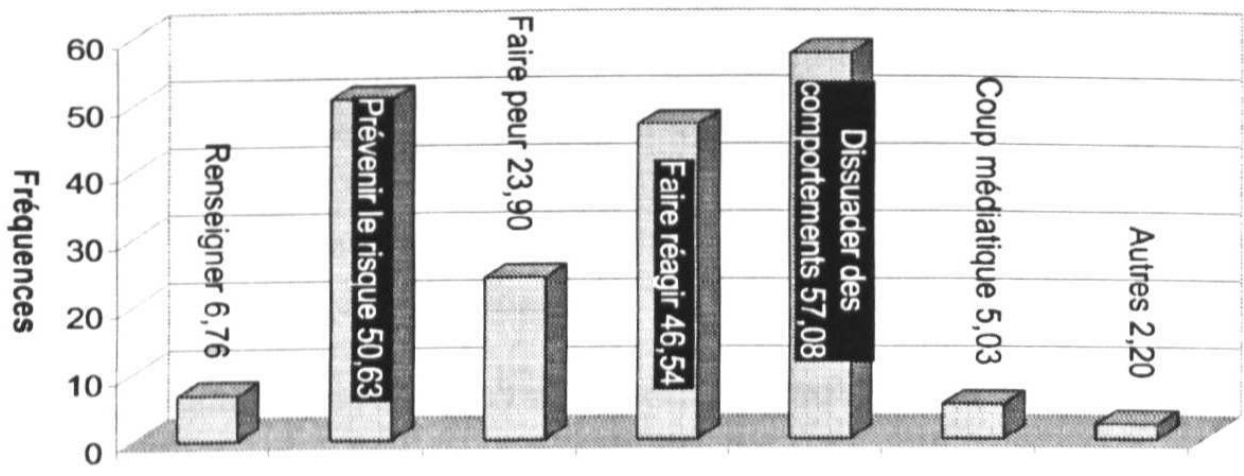

Fréquences des objectifs attribués à l'opération par les usagers

L'analyse des résultats montre que globalement les usagers ont bien identifié les objectifs de sécurité routière (dissuader, prévenir, faire réagir). En effet, tous les objectifs de sécurité routière appartiennent aux quatre objectifs majoritaires énoncés (« prévenir le risque » $50,6 \%$, «faire peur » $23,9 \%$, «faire réagir » $46,5 \%$, «dissuader des comportements » $57,1 \%$ ).

Nous observons une perception des objectifs chez les plus de 64 ans de qualité moindre. En effet, pour cette population, les objectifs de « coup médiatique », « renseigner », « faire peur » ont des fréquences équivalentes et supérieures à l'objectif «faire réagir». Ces usagers apparaissent comme plus résignés que leurs cadets. Cet état notifie un sentiment d'impuissance, de fatalité. Faut-il lier ces observations à une difficulté chez ces personnes à s'adapter aux nouvelles conditions de trafic? Des études complémentaires pourraient en préciser le sens. 
79 Les organisations, auxquelles les usagers attribuent l'initiative de l'opération « silhouette ", se répartissent comme suit :

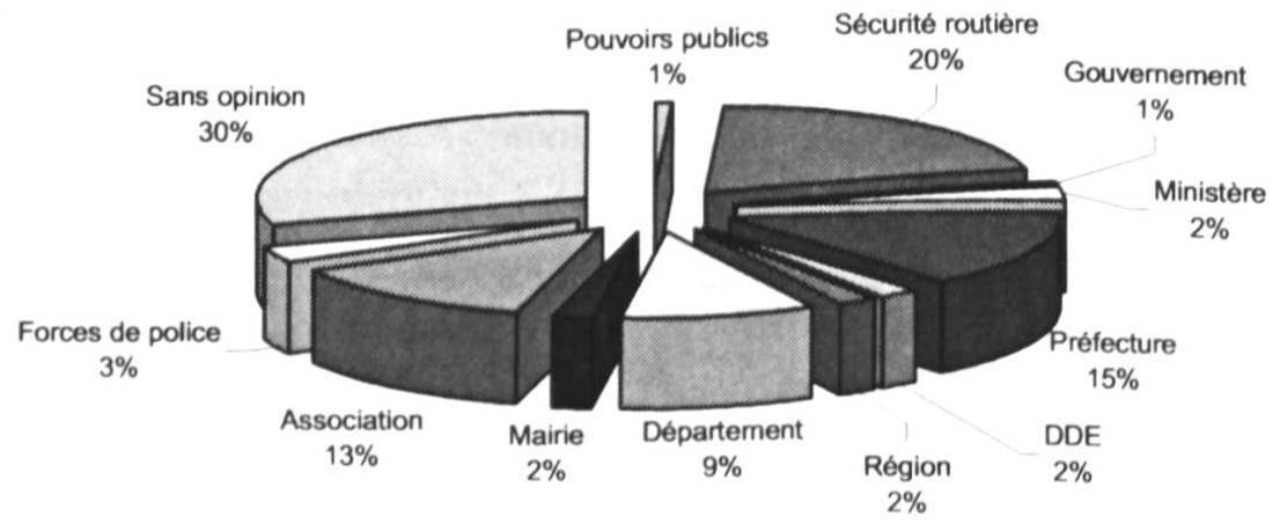

Répartition des organisateurs de l'opération attribués par les usagers

Fréquences des organismes attribués par les usagers comme initiateur de l'opération «silhouette» :

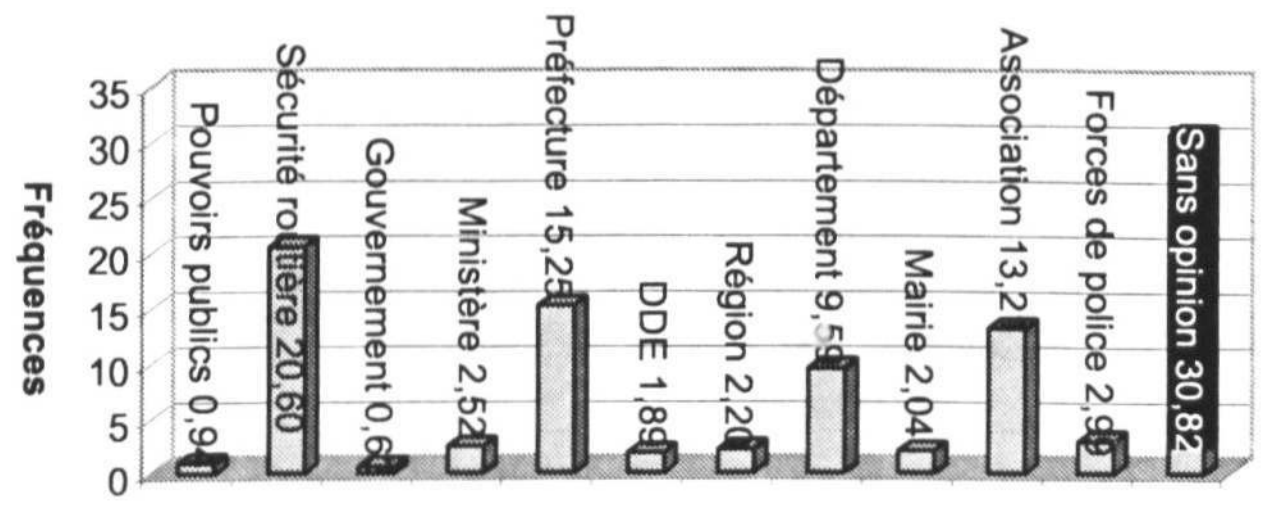

Fréquences des organisateurs de l'opération attribués par les usagers "sécurité routière » 20,6\%. Or, la verbalisation "sécurité routière " ne peut-être clairement identifiée comme exprimant l'organisme préfectoral de sécurité routière (Mission de sécurité routière). Globalement, les usagers reconnaissent mal la structure qui organise l'opération ( $1 / 3$ des usagers n'ont pas d'opinion). Ces résultats peuvent surprendre car sur chaque axe routier un panneau annonce, d'une part, les silhouettes disposées sur cet axe et, d'autre part, l'organisme qui en a pris l'initiative, ici, en l'occurrence la préfecture. Ceci montre que, pour l'usager, ce qui fait sens, c'est bien la position des silhouettes et ce qu'elles symbolisent. Toutes autres informations n'ont pas de valeur dans l'activité "conduire» ou ne sont pas identifiées comme pouvant être valorisables dans cette activité. Vulgairement, pour l'usager ce qui est important ce sont les silhouettes, l'organisateur n'a aucune importance.

Les jugements d'intérêts portés par les usagers sur l'opération «silhouette» se répartissent comme suit : 


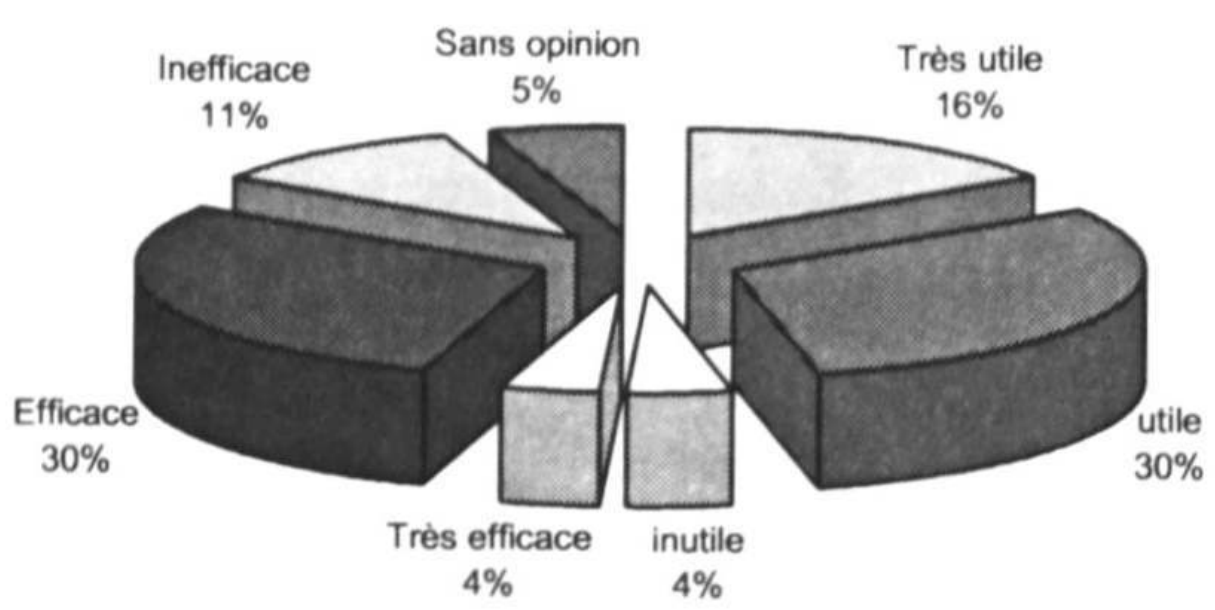

Répartition des jugements d'intérêts verbalisés par les usagers

Fréquences des jugements d'intérêts portés sur l'opération « silhouette » :

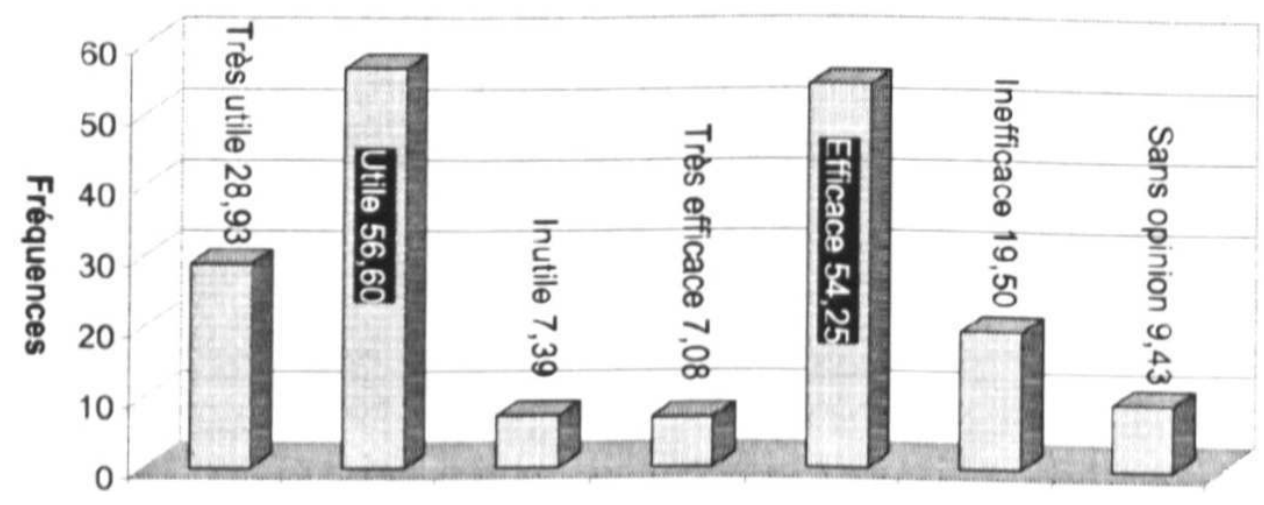

Fréquences des jugements d'intérêts des usagers

Globalement, ces résultats montrent que : 85,5\% des usagers jugent cette action utile. $61,3 \%$ des usagers estiment cette action efficace. $75 \%$ des usagers attendent qu'un travail d'explication soit poursuivi.

Plus d'hommes $(21 \%)$ que de femmes (16\%) trouvent cette opération inefficace. Les usagers âgés de plus 64 ans (30\%) sont proportionnellement plus nombreux que leurs cadets ( $21 \%$ chez les moins 25 ans, $18 \%$ chez les 25-64 ans) à estimer cette opération « inefficace ». Les usagers de plus de 64 ans (23\%) sont plus nombreux que leurs cadets à juger cette opération « inutile ». Ces observations sont en cohérence avec les observations de l'analyse des objectifs attribués par les usagers. En effet, nous avons écrit précédemment que les usagers de 65 ans et plus apparaissaient comme plus résignés, fatalistes. Le fait qu'ils sont plus enclins à juger l'opération d'inefficace et d'inutile correspond bien \un aveu d'impuissance et de résignation devant la fatalité.

Le fait que $75 \%$ des usagers attendent qu'un travail d'explication soit poursuivi montre que si l'usager réalise bien le lien entre ces silhouettes et le discours de sécurité routière, il est en attente d'information car il ne sait pas comment investir ce discours ou s'il l'investit bien. Que fait-on de l'information portée par la silhouette? 


\section{Conclusion}

Les résultats montrent qu'il s'agit d'un bon outil de communication qui ne choque pas l'usager. Si l'usager réalise bien le lien entre les silhouettes et le discours de sécurité routière, il est en attente d'information, car il ne sait pas comment investir ce discours ou s'il l'investit bien. Que fait-on de l'information portée par la silhouette?

Quand l'usager rencontre les silhouettes, il s'intéresse plus à son activité de déplacement, il est plus attentif et va jusqu'à ralentir. La verbalisation, acte de verbaliser les effets induits par les silhouettes, permet à l'usager d'en prendre conscience et de qualifier cette opération d'utile et d'efficace. Le fait qu'un usager communique sur cette opération à sept autres usagers (en moyenne) participe à un travail de prise de conscience collective.

9 L'usager se représente mal l'organisation qui a porté cette action. Un travail de communication spécifique pourrait être entrepris pour clarifier la perception du domaine « sécurité routière ».

Les erreurs de localisation des silhouettes peuvent induire un excès de confiance sur le réseau non équipé. Une action en direction du réseau non équipé serait souhaitable. L'extension de cette opération à tout le réseau routier départemental pose la question de la banalisation.

1 Que peut-on envisager pour éviter un phénomène de banalisation ? Plusieurs actions sont envisageables :

Modifier la position des silhouettes, chaque année, en fonction de l'évolution des accidents (position des silhouettes sur les sept dernières années) ;

Enrichir progressivement l'information disponible sur la silhouette :

- Ajouter un liseré fluorescent pour les silhouettes d'accidents survenus la nuit (silhouettes qui deviennent visibles en conduite nocturne);

5 - Ajouter une information fluorescente (adresse d'un site Internet) qui permettrait à tout automobiliste d'accéder à des informations relatives aux conditions de l'accident (conditions déterminées à partir des rapports des enquêtes REAGIR).

6 Les différences observées selon l'âge et l'origine socio-professionnelle de l'usager, montrent que communiquer efficacement sur le risque routier est possible en dehors d'un discours moralisateur (discours qui touche essentiellement les personnes disposées à l'entendre) dès lors que l'on utilise des outils de communication susceptibles de symboliser un ou des éléments fondateurs ou référant au risque. Il est vrai que l'interprétation de ces symboles varie en fonction de la population concernée. Ce dernier résultat plaide en faveur d'une approche différenciée de la population. Un travail d'investigation pourrait être développé en ce sens. 


\section{BIBLIOGRAPHIE}

AUBERT, G., Le traitement des connecteurs logiques par des enfants de 10-12 ans. Thèse de doctorat, 19 janvier 1994.

AUBERT, G., Le traitement de connecteurs logiques par des enfants de 10-12 ans. In revue Enfance, $n^{\circ} 2 / 1998$, p. 189-212.

GRAS, A. et MORICOT, C. et POIROT-DELPECH, S. L et... [et al.], Face à l'automate : le pilote, le contrôleur et l'ingénieur. Paris : Publications de la Sorbonne, V-310 p. Collection : Série Homme et société, ISSN 0292-6679, 20, 1994.

JOSHUA et DUPIN, Introduction à la didactique des sciences et des mathématiques. PUF, 1993.

KARMILOFF-SMITH, A., Beyond Modularity : a Developmental Perspective on Cognitive Science.

Cambridge, Mass. : MIT Press, 1992.

LE BRETON, D., Passions du risque. Paris : Métailié, Collection Traversées, 1991.

PONCE, C, Apprentissage différencié de l'ordination de nombres rationnels à l'aide d'un tutoriel. Thèse de doctorat, juin 1993.

PONCE, C, Pédagogie différenciée. In revue Française de Pédagogie, $\mathrm{n}^{\circ}$ 114, janvier-février-mars 1996, p. 97-102.

PONCE, C, Contraintes d'apprentissage liées aux connaissances préalables des apprenants. In Lire, écrire, compter, apprendre, Foulin, JN \& Ponce, C, avril 2000.

\section{NOTES}

1. Une conception est une représentation qui remplit trois conditions : communauté, efficacité et stabilité.

2. Association Spirale : association de peintre et de sculpteurs, l'opération a été initiée par J.P. Giraud, sculpteur et père de victime.

3. Unité de recherche associée, CNRS EP1594, Langage et Cognition (LaCo) dirigée par M. le Professeur Daniel GAONAC'H

\section{RÉSUMÉS}

Cette étude propose d'articuler un modèle sociologique de la prise de risque et un modèle psychologique de l'homme afin de définir des critères pour choisir un outil de communication concernant le risque routier. L'outil retenu (une silhouette noire) a été évalué sur sa qualité communicative et sur celle des contenus de communication évoqués chez l'usager. Cette 
évaluation (sur une population de 636 usagers) a montré que la silhouette comme symbole de la mort est un outil de communication efficace en sécurité routière.

In order to define criteria to choose the adequate mean of communication about road risk, we suggest to link a sociological model of risk taking and a human psychological model. The selected tool (a black silhouette) has been assessed about a communicative quality and a quality of the contents that this tool suggest to people. That assesment (about 636 road users) showed that black figures used as symbols of death are an effective mean of communication as concern the road safety.

\section{INDEX}

Mots-clés : risque, qualité, évaluation, silhouette, sécurité routière, mort

\section{AUTEUR}

\section{GEORGES AUBERT}

Docteur en psychologie et sciences de l'éducation. Ingénieur de recherche contractuel du CNRS à l'unité de recherche associée, CNRS EP1594, Langage et Cognition (LaCo) Poitiers. Membre de l'équipe de psychologie du développement et de l'éducation au laboratoire de psychologie de l'université Victor Ségalen Bordeaux2. Les thèmes de recherches : le raisonnement humain, l'apprentissage des règles linguistiques, l'apprentissage de la chaîne numérique, psychologie des organisations, le risque routier chez l'enfant, l'adolescent, et l'adulte. 